\title{
A Novel Algorithmic Diagnostic Approach to Secretory Carcinoma of Salivary Gland
}

\author{
Shubhada $\mathrm{K}^{1^{*}}$, Oza $\mathbf{N}^{2}$, Patil $\mathbf{A}^{1}$, Bal $\mathbf{M}^{1}$, Pai $\mathbf{T}^{1}$, Gupta $\mathbf{R}^{3}$ and Chaturvedi $\mathbf{P}^{4}$
}

${ }^{1}$ Department of Pathology, Tata Memorial Hospital, Dr. E. Borges Road, Parel, Mumbai, Maharashtra, India

${ }^{2}$ Department of Pathology, Mazumdar Shaw Medical Centre, Narayana Hrudralaya Health City, Bangalore, Karnataka, India

${ }^{3}$ Department of Tissue Pathology and Diagnostic Oncology, Central Clinical School, University of Sydney, Sydney, NSW, Australia

${ }^{4}$ Head and Neck Oncology, Tata Memorial Hospital, Dr. E. Borges Road, Parel, Mumbai, Maharashtra, India

"Corresponding author: Shubhada K, Department of Pathology, Tata Memorial Hospital, Dr. E. Borges Road, Parel, Mumbai-400012, Maharashtra, India, Tel: +91-9223424134; E-mail: drsvkane@gmail.com

Received date: May 30, 2018; Accepted date: July 23, 2018; Published date: July 27, 2018

Copyright: (c) 2018 Shubhada K, et al. This is an open-access article distributed under the terms of the Creative Commons Attribution License, which permits unrestricted use, distribution, and reproduction in any medium, provided the original author and source are credited.

\begin{abstract}
Background: Secretory carcinoma (SC) is a recently recognized tumour of salivary gland with characteristic $\mathrm{t}$ $(12 ; 15)$ (q13; q25) translocation with ETV6-NTRK3 fusion. SC were misdiagnosed as Acinic cell carcinoma (AciCC), especially Papillary cystic variant (PCV) in the past. Primary objective of the study was to devise diagnostic algorithm to distinguish SC from other low grade salivary gland tumors especially AciCC.

Methods: Surgical pathology archives was searched for cases diagnosed as PCV-AciCC from 2005 to 2017 and as SC from 2012-2017. The H\&E, IHC and FISH results were studied.

Results: Parotid and oral cavity were involved in $74.3 \%$ and $14.2 \%$ cases. H\&E sections showed predominant papillary-cystic, microcystic and solid pattern in $60 \%, 31.4 \%$ and $8.6 \%$ cases. Hob-nailing and cytoplasmic multivacuolation was seen in $68.6 \%$ and $91.4 \%$ cases. We identified 3 low power indicators of SC: a) papillae lined by hobnail cells; b) solid pattern showing multivacuolated bubbly cytoplasm; c) follicular pattern with dense colloid like secretions. A diagnostic algorithm was devised. Of 35 cases, upfront diagnosis of SC was offered in 22 cases. 13 Cases of PCV-AciCC were reclassified as SC based on morphology and confirmed by IHC (diffuse co-expression of mammaglobin, S100 and lack of DOG1 positivity) and molecular study. $65.7 \%$ cases showed ETV6 translocation by FISH.
\end{abstract}

Conclusion: SC is a new entity, which was misdiagnosed as PCV-AciCC in the past. SC can originate in minor salivary gland. Awareness of morphological indicators and high index of suspicion is necessary for diagnosis. IHC markers further facilitate the diagnosis. The translocation study can thus be limited to cases with unusual histology and planning of targeted therapy in future. A novel diagnostic algorithm is suggested for recognition of this new entity.

Keywords: Secretory carcinoma; Papillary cystic variant; Acinic cell carcinoma; Hobnail cells; Cytoplasmic multivacuolation; ETV6 translocation

\section{Introduction}

Mammary analog secretory carcinoma of salivary gland origin is a recently described tumor that harbors a characteristic balanced $t(12$; 15) (p13; q25) chromosomal translocation resulting in an ETV6NTRK3 fusion identical to that commonly found in secretory carcinoma (SC) of the breast [1,2]. Since the initial description in 2010 [1], MASC has been increasingly recognized and more than 150 cases have already been published in the English literature from different countries [3]. The most recent version of the World Health Organization Classification of Head and Neck Tumors, however, utilizes the terminology "secretory carcinoma" for consistency as SCs have been recently described at other extra salivary and extra mammary sites, such as thyroid gland, skin, and sinonasal mucosa $[2,4]$.
Histologically, SC is characterized by uniform cells with blandlooking vesicular round nuclei, distinct nucleoli and eosinophilic multivacuolated cytoplasm, arranged in microcystic, papillary, follicular and solid patterns [5]. One of the diagnostic hallmarks is presence of colloid-like secretory material which fills the lumina [1].

The close differential diagnosis of SC in salivary gland is Acinic cell carcinoma (AciCC) which accounts for $12-17 \%$ of primary salivary gland neoplasm. Both tumors share common architectural patterns and cytomorphology. AciCC is characterized by zymogen granules in the cytoplasm; however, the zymogen granule poor AciCC is indistinguishable from SC [2,6-8]. Since, the entity of SC was defined in 2010 [1]; most cases were thus diagnosed as AciCC before that. The primary objectives of this study were a) to devise a diagnostic algorithm to distinguish SC from other low grade SGT especially AciCC, b) to assess whether PCV-AciCC were misdiagnosed as SC in past. 


\section{Materials and Methods}

The surgical pathology archives of a tertiary referral cancer centre were searched for all cases diagnosed as PCV-AciCC from 2005 to 2017 and those reported upfront as SC from 2012-2017. The electronic medical records were reviewed for relevant demographic and clinical information.

\section{Histology and immunohistochemistry (IHC)}

The corresponding Hematoxylin and Eosin (H\&E), special stains (PAS, PAS D, Mucin) and IHC sections of all cases were retrieved and studied. Minimum of three tumor blocks were analysed for each case and the following histologic features were recorded: (1) tumour border, (2) histologic patterns, (3) nuclear contours, (4) nuclear size compared with small lymphocytes, (5) chromatin patterns (vesicular or condensed), (6) distinct nucleoli (7) presence of vacuolated cytoplasm, (8) presence of cytoplasmic basophilic granules, (9) secretions or mucin within microcystic or tubular spaces, (10) presence of hobnail cells, (11) mitotic figures and degree of necrosis, (12) degree of hemorrhage or hemosiderin deposition, (13) presence of perineural or lymphovascular invasion and (14) Mucin, PAS with and without diastase staining.

The unstained five micron sections prepared from FFPE tissues were used for special stains for PAS, PAS-D and mucin. Immunohistochemistry (IHC) was performed using Ventana benchmark XT automatic stainer. All the cases were stained with antibodies using standard polymer technique as shown in Table 1.

\begin{tabular}{|l|l|l|l|l|}
\hline Antibody specificity & Clone & Dilution & Source & Staining pattern \\
\hline Mammoglobin & 31A5 & $1: 50$ & Cell Marque, USA & Membranous \\
\hline CK7 & OV-TL 12/30 & 0.388889 & Dako, Denmark & Membranous \\
\hline S100 Protein & Polyclonal & 1.291667 & Dako, Denmark & Nuclear and cytoplasmic \\
\hline Vimentin & VG & 0.458333 & Dako & Cytoplasmic \\
\hline P63 & BC4A4 & 0.180556 & Biocare, California & Nuclear \\
\hline GCDFP-15 & LGC3FP & 0.180556 & Novocastra, Newcastle & Cytoplasmic \\
\hline MUC-4 & Mouse monoclonal & 0.736111 & Abcam, Cambridge USA & Cytoplasmic \\
\hline DOG1 & DOG1.1 & 0.09375 & Leica, Newcastle, UK & Membranous and cytoplasmic \\
\hline SOX10 & BC34 & $1: 50$ & Biocare, California, USA & Nuclear \\
\hline
\end{tabular}

Table 1: Antibodies used for immunohistochemical study.

\section{Fluorescence in situ hybridization (FISH) analysis}

The $\mathrm{t}(12 ; 15)$ (p13; q25) in SC results in ETV6-NTRK3 fusion gene product which is confirmed with the rearrangement of the ETV6 gene by FISH. Tissue microarrays were prepared with manual tissue microarrayer with 2.0 millimeter diameter tumor tissues from the selected block.

Standard procedure was followed by using approximately $10 \mu \mathrm{l}$ of Commercial ZytoLight SPEC ETV6 Dual Color Break Apart Probe (Zytovision, Bremerhaven, Germany). The slides were scored using an Olympus BX53F upright fluorescence microscope equipped with appropriate excitation and emission filters, QIcam (Q34130) Olympus camera and Qcapture pro 7.0 image analyser software. At least 100 non-overlapping tumor cell nuclei were counted. When more than $15 \%$ tumor cells demonstrated split (break-apart) signals, the case was classified as positive for ETV6 gene rearrangement $[3,8]$.

\section{Results}

This study accessed the archives and retrieved 22 cases of salivary gland carcinoma reported between 2012-2017 with upfront diagnosis of SC on morphology and IHC and later confirmed by FISH test. We also retrieved 25 cases diagnosed as AciCC-PCV from 2005-2017. Of these 25 cases, 13 cases were reclassified on review as SC on the basis of lack of zymogen granules and immunopositivity for Mammaglobin, S100 protein and immunonegativity for DOG1. Two cases retained the diagnosis of AciCC as they showed focal secretory granules, DOG1 positivity and mammaglobin negativity. Ten cases could not be included in the study due to lack of adequate material. Possible reasons could be a) poor primary tissue preservation b) suboptimal processing of old archival/outside referred paraffin blocks, c) depletion of the tumor tissue after recuts and IHC. The study thus included 35 cases of SC.

\begin{tabular}{|l|l|l|l|l|l|}
\hline Age (years) & Sex & Location & Size (cms) & Pathologic Stage & Follow up \\
\hline $\begin{array}{l}\text { Median age } \\
33\end{array}$ & Male 18 & Parotid 26 (74.3\%) & Mean 3 3 3 & T1 09 (25.7\%) & Median 12 months \\
\hline & & & & T2 16 (45.7\%) & \\
\hline Range 09-66 & Female 17 & Sub-mandibular gland 04 (11.4\%) & $\begin{array}{l}\text { Range } \\
01-06\end{array}$ & T3 06 (17.1\%) & Range 03-156 months \\
\hline
\end{tabular}


Citation: Shubhada K, Oza N, Patil A, Bal M, Pai T, et al. (2018) A Novel Algorithmic Diagnostic Approach to Secretory Carcinoma of Salivary Gland. J Cytol Histol 9: 511. doi:10.4172/2157-7099.1000511

Page 3 of 8

\begin{tabular}{|l|l|l|l|l|l|}
\hline & $\begin{array}{l}\text { Male: female ratio } \\
1.1: 1\end{array}$ & Minor salivary gland (Alveolus, hard palate) $05(14.2 \%)$ & T4 04 (11.4\%) & Died of Disease 00 \\
\hline & & & & $\begin{array}{l}\text { Local } \\
(17.14 \%)\end{array}$ & recurrence 06 \\
\hline & & & & $\begin{array}{l}\text { Node Metastasis 07 (20\%) } \\
(82.3 \%)\end{array}$ \\
\hline & & & & evidence of disease 28 \\
\hline
\end{tabular}

Table 2: Salient clinical findings in Secretory Carcinoma $(\mathrm{n}=35)$.

\begin{tabular}{|l|l|l|l|}
\hline Predominant Patterns & Re-classified $(\mathbf{n = 1 3 )}$ & Upfront cases (n=22) \\
\hline Microcystic & $2(15.3 \%)$ & $9(40.9 \%)$ & $11(31.4 \%)$ \\
\hline Solid & $1(7.6 \%)$ & $2(9.1 \%)$ & $3(8.6 \%)$ \\
\hline Papillary & $10(76.9 \%)$ & $11(50 \%)$ & $21(60 \%)$ \\
\hline
\end{tabular}

Table 3: Histomorphological patterns in reclassified and upfront cases of SC.

\begin{tabular}{|c|c|}
\hline Histopathological parameters & $\mathrm{N}=35(\%)$ \\
\hline \multicolumn{2}{|l|}{ Predominant Pattern } \\
\hline Microcystic & $11 / 35(31.4 \%)$ \\
\hline Solid & $03 / 35(08.6 \%)$ \\
\hline Papillary & $21 / 35(60.0 \%)$ \\
\hline Cytoplasmic vacuolation & $32 / 35(91.4 \%)$ \\
\hline Hobnailing & $24 / 35(68.6 \%)$ \\
\hline \multicolumn{2}{|l|}{ Nuclear shape } \\
\hline Round & $32 / 35(91.4 \%)$ \\
\hline Irregular & $03 / 35(08.6 \%)$ \\
\hline \multicolumn{2}{|l|}{ Nucleoli: } \\
\hline Prominent & $32 / 35(91.4 \%)$ \\
\hline Inconspicuous & $03 / 35(08.6 \%)$ \\
\hline \multicolumn{2}{|l|}{ Chromatin } \\
\hline Vesicular & $30 / 35(85.7 \%)$ \\
\hline Condensed & $05 / 35(14.2 \%)$ \\
\hline Basophilic granules & $00 / 35$ \\
\hline Intra-cytoplasmic mucin & $27 / 35(77.1 \%)$ \\
\hline Necrosis & $00 / 35$ \\
\hline Peri-neural invasion & $02 / 35(05.7 \%)$ \\
\hline Lymphovascular invasion & $00 / 35$ \\
\hline
\end{tabular}

Table 4: Histopathological findings $(\mathrm{N}=35)$. 
Citation: Shubhada K, Oza N, Patil A, Bal M, Pai T, et al. (2018) A Novel Algorithmic Diagnostic Approach to Secretory Carcinoma of Salivary

Page 4 of 8

The salient clinical findings are summarized in Table 2. The median age of patients was 33 years with male to female ratio of 1.1:1. Parotid was most commonly involved site accounting for $74.3 \%$ cases. Minor salivary gland was involved in $5(14.3 \%)$ cases. H\&E stained sections showed a lobulated tumor divided by fibrous septa. The tumour invasive front was smooth and showed papillary, solid, follicular and microcystic patterns (Figure 1A and 1B). In most cases, these patterns were intermixed. The different morphological patterns were studied in reclassified and upfront diagnosed cases which are enumerated in Tables 3 and 4 .

The papillae were covered with single layer of cuboidal epithelium with distinct hob-nailing of cells in $68.6 \%$ (Figure 1C). The intraluminal spaces were filled with colloid like dense eosinophilic secretions which were PAS positive diastase resistant. Scalloping of the border due to resorption of colloid was noted (Figure 1D).
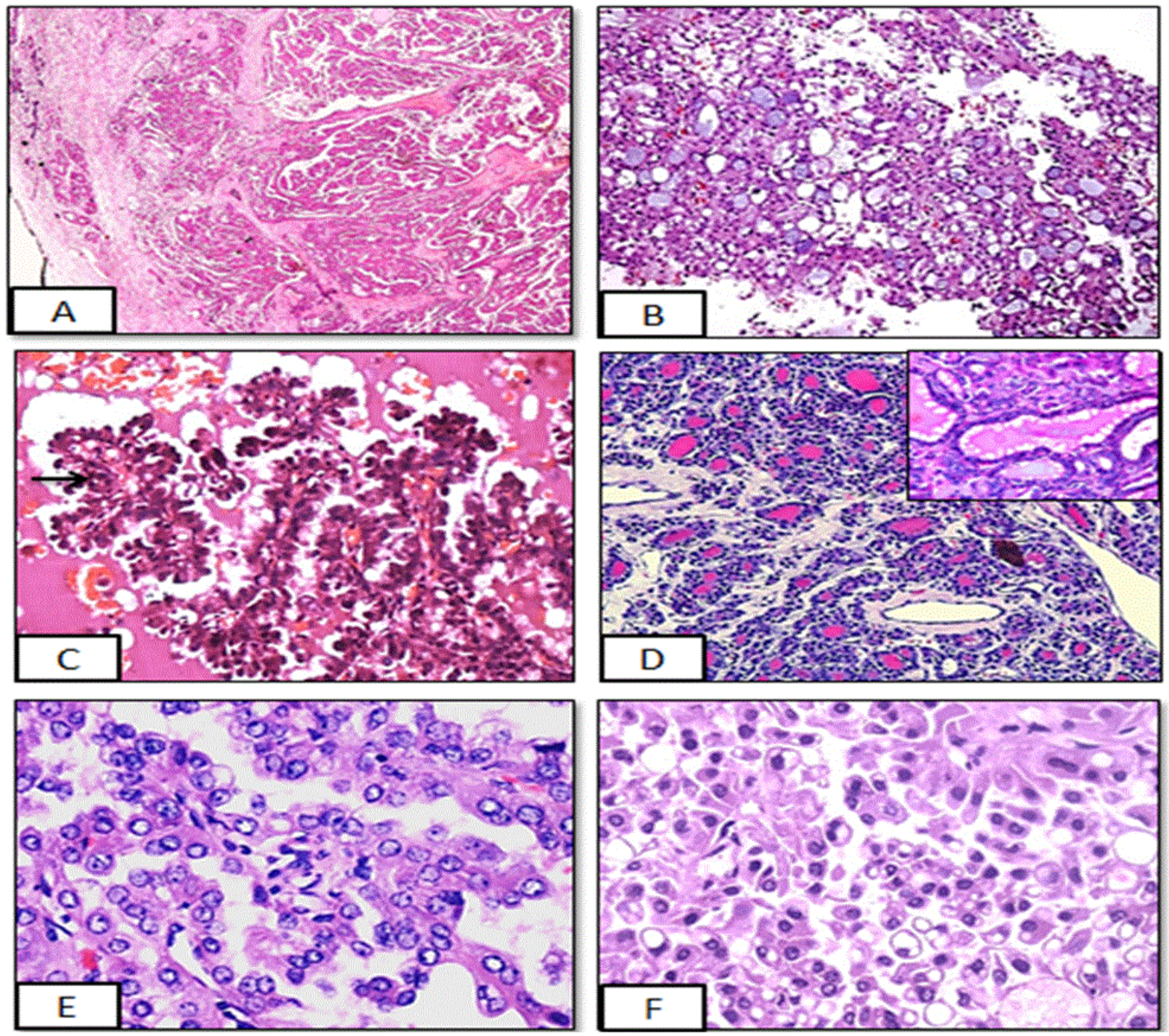

Figure 1: H\&E stained section shows a well circumscribed tumor with papillary and microcystic architecture (A and B); Hobnailing of the cells surrounding the fibrovascular core (arrow) (C); follicular pattern with dense colloid like secretions with scalloping (resorption-inset) of colloid (thyroidization) (D); Low grade vesicular nuclei with prominent nucleoli (E); Abundant bubbly eosinophilic cytoplasm (F). Original magnification: $\mathrm{x} 40(\mathrm{~A}, \mathrm{~B}) ; \mathrm{x} 200(\mathrm{C}, \mathrm{D}) ; \mathrm{x} 400(\mathrm{E}, \mathrm{F})$.

The tumor showed uniform central round nuclei, vesicular nuclear chromatin, and distinct nucleoli (Figure 1E). Cytoplasmic multivacuolation was noted in $91.4 \%$ cases. Abundant eosinophilic cytoplasm (Figure 1F) with scattered intracytoplasmic mucin globules were seen in $77.1 \%$ cases each. Focal presence of basophilic granules indicating serous differentiation was not seen in any case. Occasional mitotic figure was noted. Necrosis and lymphovascular emboli were absent in all the cases. Perineural invasion was noted in two (5.7\%) cases. High grade transformation was not seen in any of the cases. 
Citation: Shubhada K, Oza N, Patil A, Bal M, Pai T, et al. (2018) A Novel Algorithmic Diagnostic Approach to Secretory Carcinoma of Salivary Gland. J Cytol Histol 9: 511. doi:10.4172/2157-7099.1000511

Page 5 of 8

\begin{tabular}{|l|l|l|l|l|l|l|l|l|l|l|l|}
\hline No. of Cases & Mg & CK7 & S100 P & Vimentin & P63 & GCDFP & MUC-4 & SoX10 & DOG-1 & FISH Positive & FISH Un-interpretable \\
\hline $\mathrm{N}=34$ & $34(100 \%)$ & $34(100 \%)$ & $34(100 \%)$ & $34(100 \%)$ & 0 & 0 & $30(93.80 \%)$ & 0 & 0 & $22(64.70 \%)$ & $12(35.20 \%)$ \\
\hline
\end{tabular}

Table 5: Immunohistochemical and molecular study findings.

The luminal secretions showed PAS positivity with diastase resistance and Mucin positivity in $94 \%$ and $77 \%$ cases, respectively. In addition, the cytoplasm in these cases was PAS positive diastase labile. On immunohistochemistry, the tumor cells strongly expressed Mammaglobin, S100 protein, CK7 and Vimentin; while stained negative for P63, GCDFP 15, SOX10 and DOG1 (Figure 2A-2E). Strong luminal positivity for MUC 4 was noted in $85.7 \%$ cases. MUC4 was uninterpretable in $14.2 \%$ cases due to tissue depletion. 13/25 cases of previously diagnosed PCV-AciCCs were re-classified as SCs based on morphology, special stain and immunohistochemistry (Table 5).

\begin{tabular}{|l|l|l|l|l|l|l|l|}
\hline Authors & $\begin{array}{l}\text { Skalova et al. } \\
{[\mathbf{1}]}\end{array}$ & $\begin{array}{l}\text { Connor et al. } \\
{[\mathbf{1 7}]}\end{array}$ & $\begin{array}{l}\text { Bishop et al. } \\
{[\mathbf{2 5}]}\end{array}$ & $\begin{array}{l}\text { Skalova et al. } \\
{[\mathbf{3 0}]}\end{array}$ & $\begin{array}{l}\text { Majewska et al. } \\
{[\mathbf{1 2}]}\end{array}$ & $\begin{array}{l}\text { Nasir et al. } \\
{[\mathbf{2 1}]}\end{array}$ & $\begin{array}{l}\text { Present Study } \\
\mathbf{( 2 0 1 7 )}\end{array}$ \\
\hline No. of cases & 16 & 7 & 11 & 3 & 7 & 11 & 35 \\
\hline ETV6 gene rearrangement & 13 & 7 & 11 & 3 & 6 & 10 & 22 \\
\hline
\end{tabular}

Table 6: Comparison of number of cases and translocation study reported in literature.

All the cases were subjected to molecular testing. $65.7 \%(22 / 35)$ cases showed characteristic ETV6 translocation in more than $15 \%$ of the tumor cells; while $34.2 \%,(12 / 35)$ cases were reported as uninterpretable (Figure $2 \mathrm{~F}$ ) due to inadequate material. Not a single case was reported as negative for translocation (Figure 3).

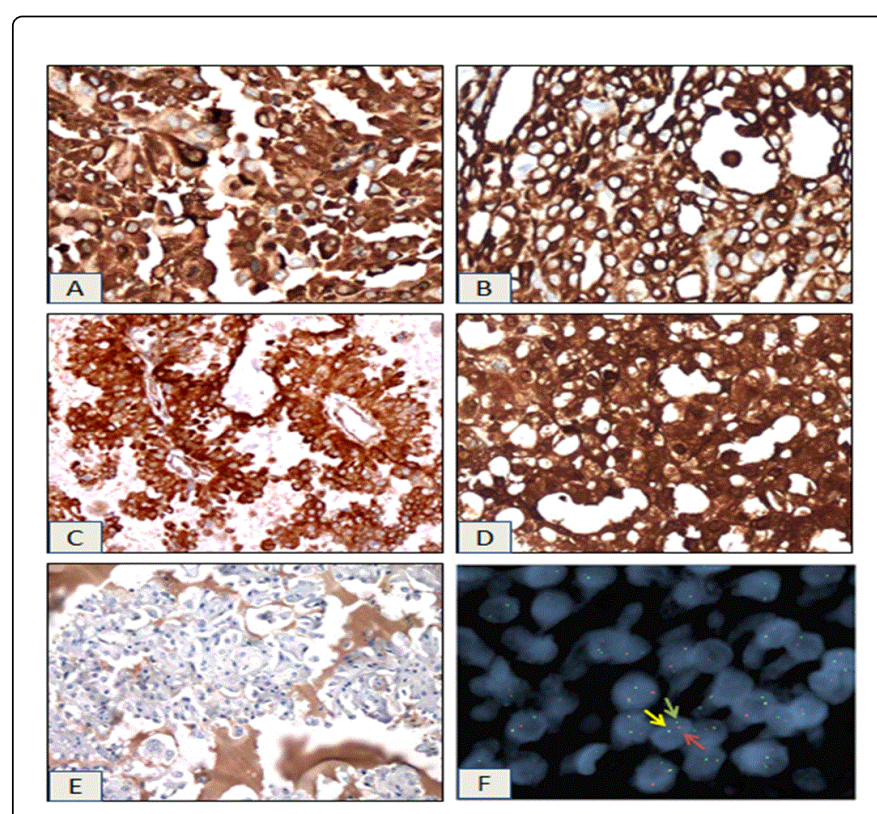

Figure 2: Tumor cells show strong positivity for Mammaglobin and CK7(membranous) (A,B); MUC4 (cytoplasmic) (C); S100 Protein (Cytoplasmic and nuclear) (D); Negative for DOG1 (E); ETV6 split signal positivity seen by FISH (F) Original magnification: $\mathrm{x} 400$ $(\mathrm{A}, \mathrm{B}, \mathrm{D}, \mathrm{F}) ; \mathrm{X} 200(\mathrm{C}, \mathrm{E})$.

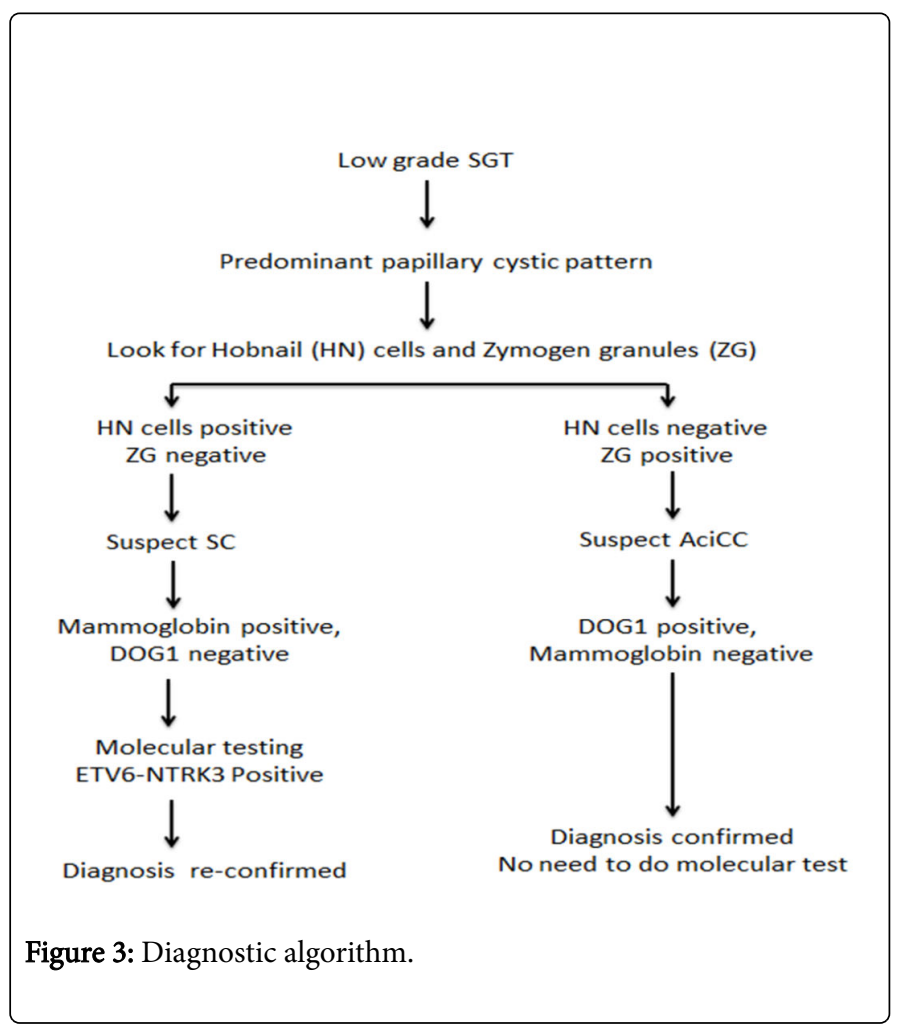

\section{Discussion}

SC is a relatively newly described entity of low grade carcinoma of salivary glands characterized in most cases by a distinctive molecular alteration: $\mathrm{t}(12 ; 15)$ (p13; q25) chromosomal rearrangement resulting in the fusion of the ETV6 and NTRK3 genes [1,5]. Interestingly, the same translocation ETV6-NTRK3 can be seen not only in SC of breast [1], but also in infantile fibrosarcoma [9] congenital mesoblastic nephroma [10] and hematopoietic malignancies [11]. However, this specific translocation is not observed in any other SGT [12]. Thus, it is tumor defining translocation. 
The true incidence of SC is currently unknown due to its rarity unfamiliarity, unavailability of molecular test and possible wrong interpretation of this tumor type. Luk et al. [13] reported 9 MASC cases in a review of 190 malignant SGT $(\sim 4.5 \%)$; whereas Majewska et al. [12] reported 7 MASC cases out of $183(\sim 4 \%)$ in a similar study. In our study, SC accounted for $1.4 \%$ of all the cases diagnosed as low grade SGT at tertiary cancer centre.

In most studies of SC, slight male predominance is noted (1.3 to 1.5$)$ [14]; similar to our study (1.1:1). Secretory carcinoma occurs predominantly in younger males as compared to AciCC which predominantly occurs in elderly females $[1,14]$. Recent articles claimed the possibility of preoperative cytological diagnosis of SC based on cytologic features aided by immunocytochemistry [15]. High index of suspicion on cytology would facilitate the FISH analysis on smears or cell blocks confirming the diagnosis and obviating the need for biopsy.

Histologically, SC is predominantly well circumscribed lesion. Few tumours may show focal infiltration in the adjacent tissue. Architecturally, SCs show solid, microcystic, follicular, tubular, papillocystic, and cribriform patterns in varying proportions [16]. Our study demonstrates that one of the patterns may be dominating in proven cases of AciCC and SC. The solid pattern is more commonly seen in AciCC as compared to SC whereas the papillary architecture is seen more frequently in SC as compared to AciCC [17]. Microcystic and follicular pattern is seen in both AciCC and SC [16-19]. Similar findings were noted in our study.

In view of architectural overlap, we studied morphology of SC at great length and identified some low power indicators which were a) predominant papillary pattern with papillae lined by Hobnail cells; b) solid pattern showing multivacuolated bubbly cytoplasm; c) follicular pattern with dense colloid like secretions with scalloping (resorption) of secretions similar to thyroidization. Hence, it is crucial to look for presence of hobnail cells in any low grade salivary gland carcinoma showing papillary pattern and think about SC and not AciCC. Though hobnailing has been mentioned by few authors, this point is not highlighted in the literature [17].

According to World Health Organization [20,21] AciCC are heterogeneous tumors comprised of varying proportion of many cell types. These included vacuolated, glandular clear cells and oncocytes admixed with granular acinar cells. The key diagnostic feature is solid pattern and the presence of granular basophilic cytoplasm containing PAS-positive diastase resistant zymogen granules earning the name of "blue dot tumor" $[16,21,22]$ and cytologic heterogenecity which is indicative of AciCC than SC.

In this retrospective study, 13 out of 25 cases earlier reported as papillary cystic variant of AciCC were reclassified as SC after IHC and FISH analysis on FFPE. The papillary pattern was also dominant in the upfront diagnosed cases of SC. Therefore, the belief that AciCCs are characterized by five basic histologic patterns i.e. solid, trabecular, papillary, follicular and microcystic needs to be re-evaluated $[16,21]$.

Cystic spaces are common in both AciCC and SCs. However, the periphery of the cysts can provide diagnostic clue [23]. We observed that most SCs including those reclassified AciCC-PCV, demonstrated interdigitating papillary projections into the cystic cavity. The papillae were lined by cells showing hobnailing of the nuclei at places. On the other hand, the cysts in AciCC lacked papillary projections, hobnailing and showed smooth inner surface. The cyst was filled with haemorrhage or basophilic secretions [13-16]. Similar findings were noted by Hsieh et al. [16] in their study. Follicular pattern with dense colloid like secretions (thyroidization) was another pattern characteristically noted in SC in our study. This was earlier mistaken for AciCC. The low power morphologic indicators should alert the pathologist to consider the differential diagnosis of SC and to order appropriate IHC panel. Thus, before considering the diagnosis of AciCC, SC should be considered especially at sites other than parotid [23] where papillary pattern is noted.

Low-grade salivary duct carcinoma (LGSDC) displays large ductal spaces with intraductal proliferations [24]. Nuclei of LGSDC are similar to those of SC, but the cytoplasm is not bubbly or pink, and it often contains yellow lipofuscin-like pigment [24,25]. Immunohistochemistry may not allow distinction, because LGSDC also shows strong and diffuse expression of S100 protein and mammaglobin. However, positivity with AR (androgen receptor) favors diagnosis of LGSDC over SC [25].

Mucoepidermoid carcinoma (MEC) is usually not difficult to distinguish from SC. However, given that SC can show focal intracytoplasmic mucin, macrocystic variants of SC may on occasion mimic cystic low grade MEC especially when these originate in minor salivary glands [26]. The important diagnostic clues here are characteristic lush papillary pattern, hobnailing and multivacuolated mucin negative cells in SC. However, the goblet cells in MEC may express mammaglobin and SC may show focal p63 expression. In such problematic cases, identifying a CRTC1-MAML2 fusion and ETV6NTRK3 fusion would be diagnostic of low grade MEC and SC, respectively $[20,26]$.

Yet another tumour with which SC can be confused at minor salivary gland is polymorphous adenocarcinoma (PA). The mucohyaline matrix seen at low-power field and presence of a variety of patterns in every section, usually allows for the quick elimination of SC [27]. PA not uncommonly co expresses S100 protein and mammaglobin, though focally. Thereby, it could easily enter into a differential diagnosis with SC in small biopsy specimens. In such a scenario, ETV6 gene rearrangement study would be necessary for its distinction.

Though many studies have described morphological features and differential diagnosis of SC at great length, none has attempted to devise a diagnostic algorithm for screening of low grade SGT on morphology [28]. Our study has thereby, illustrated a novel diagnostic algorithm that will be useful in low resource settings where molecular testing is neither easily available nor affordable (Figure 3). Comprehensive sub typing of histologic features may provide a useful screening method to detect minimum number of cases that would warrant additional IHC and molecular tests for confirmation of diagnosis.

Our study included five SC cases originating in the oral cavity which were initially diagnosed as either AciCC or MEC. Awareness of origin of SC in minor salivary gland is necessary while evaluating low grade SGT in biopsy. This particular point is not highlighted in any other studies, but referred to only in the form of case reports [29].

We believe that the long standing practice of classifying AciCCs solely on the basis of architectural growth patterns including papillary cystic in the absence of clear cut zymogenic granules in the cytoplasm is not prudent, especially after new entity of SC has been introduced into the literature $[7,18]$. SC is a distinctive SGT that is now well entrenched in the literature and needs to be entertained more frequently in the clinical practice. Currently, positive molecular test is to be considered the gold standard for the diagnosis of SC, but there is 
growing body of evidence that in future majority (up to 95\%) of tumors can be accurately classified as SC based solely on morphology and immunohistochemistry [23]. We have devised a new diagnostic algorithm which substantiates the same findings.

Skálová et al. [1] reported strong Vimentin and S100 Protein positivity in all the cases of SC. Chiosea et al. [19] reported similar findings and used expression of S100 Protein to divide patients of low grade SGT into two groups with either diffuse (53.3\% of cases) or focal staining ( $46.7 \%$ of cases). In contrast to SC, less than one-third of AciCC cases showed focal positive staining for these antigens. In the present study, staining for Mammaglobin, CK7, S100 protein was diffusely positive in $100 \%$ of SC.

In our study, lymph node metastasis was found in $7(20 \%)$ cases of SC including upfront and reclassified cases which were limited to stage T2 and T3. Only one (2.8\%) case with upfront diagnosis of SC showed nodal metastasis at presentation. We could not correlate presence of metastases with any standard histological parameters including predominant papillary pattern as the number of cases were small. Distant metastases were not seen. Local recurrence was observed in $17.1 \%$ only. These findings may be attributed to less number of years of follow up in upfront diagnosed cases (1-6 years).

Chiosea et al. [19] noted a trend towards increased lymph node metastases [22.2\%] in SC as compared to AciCC. Survival analysis also revealed a mean disease-free survival (DFS) for patients with SC of 92 months, compared with a mean DFS of 121 months for patients with AciCC but the trend was not statistically significant. Not all SC have a good prognosis and three patients reported high-grade transformation followed by an accelerated clinical course and poor outcome [30]. However it is too early to stamp the biological behavior of SC $[4,30]$. Skálová et al. in 2016 reported that SC cases with ETV6-X fusion were associated with invasive histology and an aggressive course. Hence it is necessary to distinguish AciCC from SC at the outset followed up for longer period to document biological behavior in both the tumours $[3,16]$.

In summary, detailed study of the histologic patterns of closely mimicking SGT thus revealed contrary to the old belief that papillarycystic pattern is a major growth pattern of SC and not AciCC. Thus, identification of appropriate patterns and more importantly, cell types such as hob-nailing should lead to suspicion of SC on morphology followed by the appropriate panel of immunomarkers. An ideal IHC panel would depend on the differential diagnosis considered in a given case. However, CK7, S100, Mammaglobin and DOG1 should be ordered in every suspected case of SC.

Recognizing SC and testing for ETV6 rearrangement for confirmation may be of potential value in personalized treatment in the future, because the presence of the ETV6-NTRK3 translocation represents a therapeutic target in SC. Recent studies suggested that the inhibition of ETV6-NTRK3 activation could serve as a therapeutic target for the treatment of patients using pan-TRK inhibitor entrectinib (Ignyta) with fusion at other sites [27,31]. Recently the alternative ETV6-RET transcription has been reported for treatment of those SCs with uncontrolled regional growth or SCs with metastatic foci, as treatment with entrectinib and similar drugs with the same target specificity will probably be ineffective in these SCs with alternative fusion transcript different from ETV6-NTRK. Comparison with similar study has been illustrated in Table 6 .

Unavailability of ETV6-NTRK3 translocation test in majority of the routine surgical pathology laboratories raises the question as to whether the diagnosis of SC can be attained on the basis of immunohistochemistry alone in the right setting.

Shah et al. [23] postulated that the morphologic features together with supporting IHC results are sufficient for a diagnosis of SC. At the same time, a note of caution is necessary here. We believe that the mere application of specific immunohistochemical markers as a surrogate marker for the ETV6 translocation without supportive morphologic findings is imprudent. This systematic practical approach will lead to high chances of picking up SC cases in day to day practices.

\section{Conclusion}

SC is a recently described new entity on the block of low grade SGT. SC was misdiagnosed as papillary cystic variant of AciCC in the past until more light was thrown due to various studies carried out and it became well recognized entity. There are distinct morphological and clear cut immunophenotypic differences between SC and other SGT specially AciCC. SC can originate in minor salivary gland. Awareness of these features is necessary for prompt diagnosis. A novel diagnostic algorithm will be useful in low resource setting.

Though ETV6-NTRK3 was the defining translocation to begin with, time is ripened now to diagnose SC without translocation study with the appropriate histology and immunomarkers. The use of translocation study can thus be limited in future to cases with unusual histology, better understanding the tumor biology and for planning of targeted therapy in advanced cases. Further research from different geographical locations is needed to understand the biological behavior of SC.

\section{References}

1. Skálová A, Vanecek T, Sima R (2010) Mammary analogue secretory carcinoma of salivary glands, containing the ETV6-NTRK3 fusion gene: A hitherto undescribed salivary gland tumor entity. Am J Surg Pathol 34: 599-608.

2. Skálová A, Vanecek T, Martinek P (2018) Molecular profiling of mammary analog secretory carcinoma revealed a subset of tumors harboring a novel etv6-ret translocation: report of 10 cases. Am J Surg Pathol 42: 234-246.

3. Skálová A, Vanecek T, Simpson R (2016) Mammary analogue secretory carcinoma of salivary glands: molecular analysis of 25 ETV6 gene rearranged tumors with lack of detection of classical ETV6-NTRK3 fusion transcript by standard RT-PCR: Report of 4 cases harboring ETV6-X gene fusion. Am J Surg Pathol 40: 3-13.

4. Raja S, Göran S (2017) Update from the 4th edition of the World Health Organization classification of head and neck tumors: Tumors of the salivary gland. Head and Neck Pathology 11: 55-67.

5. Todd S, Kovalovsky A, Velosa C (2015) Mammary analog secretory carcinoma, low-grade salivary duct carcinoma, and mimickers: a comparative study. Modern Pathology 28: 1084-1100.

6. Huvos AG, Paulino AFG (2004) Salivary glands. In: Mills SE (ed.) Sternberg's Diagnostic Surgical Pathology (4thedn), Lippincott Williams and Wilkins, Philadelphia, pp: 932-962.

7. Datar S, Poflee S, Pande N, Umap P (2015) Preoperative cytological diagnosis of papillary cystic variant of acinic cell carcinoma: A key consideration in patient management. J Cytol 32: 191-193.

8. Jung M, Kim S, Nam S (2015) Aspiration cytology of mammary analogue secretory carcinoma of the salivary gland. Diagn Cytopathol 43: 287-293.

9. Knezevich SR, McFadden DE, Tao W (1998) A novel ETV6-NTRK3 gene fusion in congenital fibrosarcoma. Nat Genet 18: 184-187.

10. Rubin BP, Chen CJ, Morgan TW (1998) Congenital mesoblastic nephroma $\mathrm{t}(12 ; 15)$ is associated with ETV6-NTRK3 gene fusion: 
cytogenetic and molecular relationship to congenital (infantile) fibrosarcoma. Am J Pathol 153: 1451-1458.

11. Kralik JM, Kranewitter W, Boesmueller H (2011) Characterization of a newly identified ETV6 NTRK3 fusion transcript in acute myeloid leukemia. Diagn Pathol 6: 19.

12. Majewska H, Skálová A, Stodulski D (2015) Mammary analogue secretory carcinoma of salivary glands: a new entity associated with ETV6 gene rearrangement. Virchows Arch 466: 245-254.

13. Luk PP, Selinger CI, Eviston TJ (2015) Mammary analogue secretory carcinoma: an evaluation of its clinicopathological and genetic characteristics. Pathology 47: 659-666.

14. Damjanov CI, Skenderi F, Vranic S (2016) Mammary analogue secretory carcinoma (MASC) of the salivary gland: A new tumor entity. Bosn J Basic Med Sci 16: 237-238.

15. Oza N, Sanghvi K, Shet T (2016) Mammary analogue secretory carcinoma of parotid: Is preoperative cytological diagnosis possible? Diagnostic Cytopathology 44: 519-525.

16. Hsieh M, Chou Y, Yeh S, Chang Y (2015) Papillary-cystic pattern is characteristic in mammary analogue secretory carcinomas but is rarely observed in Acinic cell carcinomas of the salivary gland. Virchows Arch 467: 145-153.

17. Connor A, Ordonez B, Shago M (2012) Mammary analogue secretory carcinoma of salivary gland origin with the ETV6 gene rearrangement by FISH: Expanded morphologic and immunohistochemical spectrum of a recently described entity. Am J Surg Pathol 36: 27-34.

18. Urano M, Nagao T, Miyabe S (2015) Characterization of mammary analogue secretory carcinoma of the salivary gland: discrimination from its mimics by the presence of the ETV6-NTRK3 translocation and novel surrogate markers. Hum Pathol 46: 94-103.

19. Chiosea SI, Griffith C, Assaad A (2012) Clinicopathological characterization of mammary analogue secretory carcinoma of salivary glands. Histopathology 61: 387-394.

20. Simpson RHW (2017) Acinic cell carcinoma. In: E-Naggar AK, Chan J, Grandis J (eds.) (4thedn), World Health Organization classification of head and neck tumors Lyons. IARC Press, France, pp: 166-167.
21. Nasir UD, Fatima S, Kayani N (2016) Mammary analogue secretory carcinoma of salivary glands: a clinicopathologic study of 11 cases. Annals of Diagnostic Pathology 22: 49-53.

22. Hanson TA (1975) Acinic cell carcinoma of the parotid salivary gland presenting as a cyst. Report of two cases. Cancer 36: 570-575.

23. Shah A, Wenig M, LeGallo D (2015) Morphology in conjunction with immunohistochemistry is sufficient for the diagnosis of mammary analogue secretory carcinoma. Head Neck Pathol 9: 85-95.

24. Brandwein-Gensler M, Hille J, Wang BY (2004) Low-grade salivary duct carcinoma: description of 16 cases. Am J Surg Pathol 28: 1040-1044.

25. Bishop JA, Yonescu R, Batista D, Begum S, Eisele DW, et al. (2013) Utility of Mammaglobin immunohistochemistry as a proxy marker for the ETV6-NTRK3 translocation in the diagnosis of salivary mammary analogue secretory carcinoma. Hum Pathol 44: 1982-1988.

26. Skálová A (2013) Mammary analogue secretory carcinoma of salivary gland origin: an update and expanded morphologic and immunohistochemical spectrum of recently described entity. Head Neck Pathol 7: S30-S36.

27. Chatura KR (2015) Polymorphous low grade adenocarcinoma. J Oral MaxillofacPathol 19: 77-82.

28. Chi HT, Ly BT, Kano Y (2012) ETV6-NTRK3 as a therapeutic target of small molecule inhibitor PKC412. Biochem Biophys Res Commun 429: 87-92.

29. Zardawi I, Hook P (2014) Mammary analogue secretory carcinoma of minor salivary glands 46: 667-669.

30. Sethi R, Kozin E, Remenschneider A (2014) Mammary analogue secretory carcinoma: Update on a new diagnosis of salivary gland malignancy. Laryngoscope 124: 188-195.

31. Skálová A, Vanecek T, Majewska H (2014) Mammary analogue secretory carcinoma of salivary glands with high-grade transformation: Report of 3 cases with the ETV6-NTRK3 gene fusion and analysis of TP53, BetaCatenin, EGFR, and CCND1 genes. Am J Surg Pathol 38: 23-33. 